\title{
CURVES WITH ZERO DERIVATIVE IN F-SPACES
}

\author{
by N. J. KALTON
}

(Received 21 June, 1979)

1. Introduction. Let $X$ be an $F$-space (complete metric linear space) and suppose $g:[0,1] \rightarrow X$ is a continuous map. Suppose that $g$ has zero derivative on $[0,1]$, i.e.

$$
g^{\prime}(t)=\lim _{h \rightarrow 0} \frac{1}{h}(g(t+h)-g(t))=0
$$

for $0 \leq t \leq 1$ (we take the left and right derivatives at the end points). Then, if $X$ is locally convex or even if it merely possesses a separating family of continuous linear functionals, we can conclude that $g$ is constant by using the Mean Value Theorem. If however $X^{*}=\{0\}$ then it may happen that $g$ is not constant; for example, let $X=L_{p}(0,1)$ $(0 \leq p<1)$ and $g(t)=1_{[0, t]}(0 \leq t \leq 1)$ (the characteristic function of $[0, t]$ ). This example is due to Rolewicz [6], [7; p. 116].

The aim of this note is to substantiate a conjecture of Rolewicz $[7, p .116]$ that every $F$-space $X$ with trivial dual admits a non-constant curve $g:[0,1] \rightarrow X$ with zero derivative. In fact we shall show, given any two points $x_{0}, x_{1} \in X$, there exists a map $g:[0,1] \rightarrow$ $X$ with $g(0)=x_{0}, g(1)=x_{1}$ and

$$
\lim _{|t-s| \rightarrow 0} \frac{g(t)-g(s)}{t-s}=0 \text { uniformly for } 0 \leq s, t \leq 1 .
$$

To establish this result we shall need to study $X$-valued martingales. Let $\mathscr{B}$ be the $\sigma$-algebra of Borel subsets of $[0,1)$ and let $\mathscr{F}_{n}(n \geq 0)$ be an increasing family of finite sub-algebras of $\mathscr{B}$. Then a sequence of functions $u_{n}:[0,1) \rightarrow X$ is an $X$-valued $F_{n}$-martingale if each $u_{n}$ is $F_{n}$-measurable and for $n \geq m$ we have $\mathscr{E}\left(u_{n} \mid \mathscr{F}_{m}\right)=u_{m}$. Here the definition of conditional expectation is the standard one with respect to Lebesgue measure $\lambda$ and there are no integration problems since each $u_{n}$ is finitely-valued.

It is easy to show that every $F$-space $X$ with trivial dual contains a non-constant martingale $\left\{u_{n}, F_{n}\right\}$ which converges to zero uniformly. However we shall need to consider dyadic martingales. Let $D_{n, k}=\left[(k-1) / 2^{n}, k / 2^{n}\right)\left(1 \leq k \leq 2^{n}, 0 \leq n<\infty\right)$. Then, for $n \geq 0$, let $\mathscr{B}_{n}$ be the sub-algebra of $\mathscr{B}$ generated by the sets $\left\{D_{n, k}: 1 \leq k \leq 2^{n}\right\}$. A dyadic martingale is simply a $\mathscr{B}_{n}$-martingale. The main point of the argument will be to show that we can find non-zero dyadic martingales which converge uniformly to zero.

We note here a connection with the recent work of Roberts [4], [5] on the existence of compact convex sets without extreme points. Indeed, in a needlepoint space (see [5]) it would be easy to show that there are non-zero dyadic martingales which converge uniformly to zero. However there are $F$-spaces with trivial dual which contain no needlepoints [2].

Glasgow Math. J. 22 (1981) 19-29. 
As usual an $F$-norm on a (real) vector space $X$ is a map $x \rightarrow\|x\|$ such that

$$
\begin{array}{cc}
\|x\|>0 \quad \text { if } & x \neq 0, \\
\|x+y\| \leq\|x\|+\|y\| & (x, y \in X), \\
\|t x\| \leq\|x\| & (|t| \leq 1), \\
\lim _{t \rightarrow 0}\|t x\|=0 & (x \in X) .
\end{array}
$$

The $F$-norm is said to be strictly concave if, for each $x \in X$ with $x \neq 0$, the map $t \rightarrow\|t x\|$ is strictly concave on $[0, \infty)$, i.e.

if $0 \leq s<t<\infty$ and $0<a, b<1$ with $a+b=1$ then, if $x \neq 0$,

$$
\|(a s+b t) x\|>a\|s x\|+b\|t x\| \text {. }
$$

Every $F$-space can be equipped with an (equivalent) $F$-norm which is strictly concave. This follows from the results of Bessaga, Petczyński and Rolewicz [1]. We may give $X$ an $F$-norm $\|\cdot\|_{0}$ so that the map $t \rightarrow\|t x\|_{0}$ is concave and strictly increasing for each $x \neq 0$. Now define $\|x\|=\|x\|_{0}^{1 / 2}$.

2. Preliminary finite-dimensional results. Suppose $N$ is a positive integer. We consider the space $\mathbb{R}^{N}$ with the natural co-ordinatewise partial ordering (i.e. $x \geq y$ if and only if $x_{i} \geq y_{i}$ for $\left.1 \leq i \leq N\right)$. We shall denote by $\left(e_{k}: 1 \leq k \leq N\right)$ the natural basis elements of $\mathbb{R}^{N}$. We shall use the idea of $\mathbb{R}^{N}$-valued submartingales and supermartingales; these have obvious meaning with respect to the ordering defined above. In addition, standard scalar convergence theorems can be applied co-ordinatewise to produce the same theorems for $\mathbb{R}^{N}$.

For $1 \leq i \leq N$, let $F_{i}$ be a continuous map $F_{i}:[0, \infty) \rightarrow[0, \infty)$ which is strictly increasing, strictly concave and satisfies $F_{i}(0)=0, F_{i}(1)=1$. Then $F_{i}$ is also subadditive since

$$
F_{i}(s) \geq \frac{s}{s+t} F_{i}(s+t) \quad(s, t>0) \text {. }
$$

Hence we may define an absolute $F$-norm on $\mathbb{R}^{N}$ by

$$
\|x\|=\sum_{i=1}^{N} F_{i}\left(\left|x_{i}\right|\right) \quad\left(x \in \mathbb{R}^{N}\right) .
$$

Now, for $x \in \mathbb{R}^{N}$, define

$$
\sigma(x)=\inf \left\{\max (\|y\|,\|z\|): x=\frac{1}{2}(y+z)\right\} .
$$

We shall need the following properties of $\sigma$.

LEMmA 2.1. (a) If $x \in \mathbb{R}^{N}$ and $x \geq 0$ then there exist $y, z \in \mathbb{R}^{N}$ with $y \geq 0, z \geq 0$, $x=\frac{1}{2}(y+z)$ and $\|y\| \leq \sigma(x),\|z\| \leq \sigma(x)$.

(b) For $x, y \in \mathbb{R}^{N}$,

$$
\begin{gathered}
|\sigma(x)-\sigma(y)| \leq\|x-y\|, \\
\sigma(x) \leq\|x\| .
\end{gathered}
$$

(c) If $x \geq 0$ and $\sigma(x)=\|x\|=1$ then, for some $k$, we have $x=e_{k}$. 
Proof. (a) is an easy consequence of a compactness argument. For (b) (2.1.1), observe that if $x=\frac{1}{2}\left(z+z^{\prime}\right)$ then

$$
y=\frac{1}{2}\left[(z+y-x)+\left(z^{\prime}+y-x\right)\right]
$$

so that $\sigma(y) \leq \sigma(x)+\|y-x\|$ and so (2.1.1) follows. (2.1.2) is an immediate consequence of the definition of $\sigma$.

We are grateful to the referee for the following short proof of (c). Suppose $x \geq 0$, $\|x\|=1, x_{i}>0$ and $x_{j}>0$ where $i \neq j$. We show $\sigma(x)<1$.

Since $F_{i}$ is concave, it has left and right derivatives at $x_{i}, \alpha_{1}$ and $\alpha_{2}$, say, with $0 \leq \alpha_{2} \leq \alpha_{1}$. Similiarly $F_{j}$ has left and right derivatives at $x_{i}, \beta_{1}$ and $\beta_{2}$ with $0<\beta_{2} \leq \beta_{1}$. For small $\mathrm{t}>0$,

$$
\begin{aligned}
\left\|x+t\left(\beta_{1} e_{i}-\alpha_{2} e_{j}\right)\right\| & <\|x\|, \\
\left\|x-t\left(\beta_{1} e_{i}-\alpha_{2} e_{j}\right)\right\| & <\|x\|+t\left(-\alpha_{1} \beta_{1}+\beta_{2} \alpha_{2}\right) \\
& \leq\|x\| .
\end{aligned}
$$

Hence $\sigma(x)<1$.

We conclude that if $\sigma(x)=1$ then $x=e_{k}$ for some $k, 1 \leq k \leq N$.

Now let

$$
\pi(x)=x_{1}+\ldots+x_{n} \quad\left(x \in \mathbb{R}^{N}\right) .
$$

TheOREM 2.2. Suppose $a \in \mathbb{R}^{N}, a \geq 0$ and $\pi(a)=1$. Then there are disjoint Borel subsets $E_{1}, \ldots, E_{N}$ of $[0,1)$ with $\lambda\left(E_{i}\right)=a_{i}(1 \leq i \leq N)$ and a scalar valued dyadic supermartingale $\theta_{n}(0 \leq n<\infty)$ such that

$$
\begin{gathered}
0 \leq \theta_{n}(t) \leq 1 \quad(0 \leq t<1,0 \leq n<\infty), \\
\lim _{n \rightarrow \infty} \theta_{n}(t)=0 \text { a.e. }
\end{gathered}
$$

and if

$$
u_{n}=\mathscr{E}\left(\sum_{i=1}^{N} 1_{E_{i}} e_{i} \mid \mathscr{B}_{n}\right) \quad(0 \leq n<\infty)
$$

then

$$
\begin{gathered}
u_{n}(t) \geq \theta_{n}(t) a \quad(0 \leq t<1,0 \leq n<\infty), \\
\left\|u_{n}(t)-\theta_{n}(t) a\right\| \leq 1 \quad(0 \leq t<1,0 \leq n<\infty) .
\end{gathered}
$$

Proof. To start observe

$$
\|a\|=\sum_{i=1}^{N} F_{i}\left(a_{i}\right) \geq \pi(a)=1 .
$$

Define $\alpha_{0}(t) \equiv \alpha_{0}$ for $0 \leq t<1$, where $0<\alpha_{0} \leq 1$ and $\left\|\alpha_{0} a\right\|=1$; then let $w_{0}(t)=\alpha_{0} a$, $0 \leq t<1$. We then define inductively sequences $\left(w_{n}: n \geq 0\right),\left(w_{n}^{*}: n \geq 1\right),\left(\alpha_{n}: n \geq 0\right)$ of 
functions on $[0,1)$, where

$$
\begin{aligned}
& w_{n}(n \geq 0) \text { and } w_{n}^{*}(n \geq 1) \text { are } \mathbb{R}^{N} \text {-valued and } \mathscr{B}_{n} \text {-measurable, } \\
& \alpha_{n}(n \geq 0) \text { is } \mathbb{R} \text {-valued and } \mathscr{B}_{n} \text {-measurable, } \\
& w_{n}(t) \geq 0 \quad(0 \leq t<1, n \geq 0), \\
& w_{n}^{*}(t) \geq 0 \quad(0 \leq t<1, n \geq 0) \text {, } \\
& \alpha_{n}(t) \geq 0 \quad(0 \leq t<1, n \geq 0), \\
& \mathscr{E}\left(w_{n+1}^{*} \mid \mathscr{B}_{n}\right)=w_{n} \quad(n \geq 0), \\
& w_{n}(t)=w_{n}^{*}(t)+\alpha_{n}(t) a \quad(0 \leq t<1, n \geq 1), \\
& \left\|w_{n}(t)\right\|=1 \quad(0 \leq t<1, n \geq 0), \\
& \left\|w_{n+1}^{*}(t)\right\| \leq \sigma\left(w_{n}(t)\right) \quad(0 \leq t<1, n \geq 0) .
\end{aligned}
$$

Indeed suppose $w_{j}, w_{j}^{*}$ and $\alpha_{j}$ have been chosen for $j \leq n$. Then

$$
w_{n}(t)=b_{n, k} \quad\left(t \in D_{n, k}\right),
$$

where $\left\|b_{n, k}\right\|=1$, and $b_{n, k} \geq 0$. Choose $y_{2 k-1}, y_{2 k} \geq 0$ so that $\max \left(\left\|y_{2 k-1}\right\|,\left\|y_{2 k}\right\|\right)=\sigma\left(b_{n, k}\right)$ and $b_{n, k}=\frac{1}{2}\left(y_{2 k-1}+y_{2 k}\right)$ (see Lemma 2.1(a)). Now define

$$
w_{n+1}^{*}(t)=y_{k} \quad\left(t \in D_{n+1, k}\right) .
$$

Then (2.2.9) and (2.2.12) are clear. Since

$$
\left\|w_{n+1}^{*}(t)\right\| \leq 1 \quad(0 \leq t<1)
$$

we can determine $\alpha_{n+1}$ to be $\mathscr{B}_{n+1}$-measurable so that $\alpha_{n+1} \geq 0$ and

$$
\left\|w_{n+1}^{*}(t)+\alpha_{n+1}(t) a\right\|=1 \quad(0 \leq t<1) .
$$

Now define

$$
w_{n+1}(t)=w_{n+1}^{*}(t)+\alpha_{n+1}(t) a \quad(0 \leq t<1)
$$

and clearly (2.2.11) holds.

Observe that

$$
\mathscr{E}\left(w_{n+1} \mid \mathscr{B}_{n}\right)=w_{n}+\mathscr{C}\left(\alpha_{n+1} \mid \mathscr{B}_{n}\right) a
$$

and if $m>n$

$$
\mathscr{E}\left(w_{n} \mid \mathscr{B}_{n}\right)=w_{n}+\left(\sum_{k=n+1}^{m} \mathscr{E}\left(\alpha_{k} \mid \mathscr{B}_{n}\right)\right) a .
$$

Hence $w_{n}$ is a submartingale and it is clearly bounded. Thus $\lim w_{n}(t)=w_{\infty}(t)$ exists almost everywhere, and $\left\|w_{\infty}(t)\right\|=1$ a.e. 
The real-valued submartingale $\left(\pi \circ w_{n}: n \geq 0\right)$ is uniformly bounded and converges to $\pi \circ w_{\infty}$ a.e. Hence

$$
\begin{aligned}
\int_{0}^{1} \pi\left(w_{\infty}(t)\right) d t & =\lim _{n \rightarrow \infty} \int_{0}^{1} \pi\left(w_{n}(t)\right) d t \\
& =\int_{0}^{1} \pi\left(w_{0}(t)\right) d t+\sum_{k=1}^{\infty} \int_{0}^{1} \alpha_{k}(t) d t
\end{aligned}
$$

by $(2.2 .13)$ since $\pi(a)=1$. Hence

$$
\int_{0}^{1} \sum_{k=1}^{\infty} \alpha_{k}(t) d t<\infty
$$

and so (a.e.) $\sum \alpha_{k}(t)<\infty$. Thus $\alpha_{n}(t) \rightarrow 0$ a.e. and $\left\|w_{n+1}(t)-w_{n+1}^{*}(t)\right\| \rightarrow 0$ a.e. Hence $\left\|w_{n+1}^{*}(t)\right\| \rightarrow 1$ and $\sigma\left(w_{n}(t)\right) \rightarrow 1$ a.e. By Lemma 2.1(b), $\sigma$ is continuous and so (a.e.)

$$
\sigma\left(w_{\infty}(t)\right)=\left\|w_{\infty}(t)\right\|=1 \text {. }
$$

As $w_{\infty}(t) \geq 0$, we conclude that

$$
w_{\infty}(t)=\sum_{i=1}^{N} 1_{E_{i}} e_{i} \quad \text { a.e. },
$$

where $E_{1}, \ldots, E_{N}$ are disjoint Borel sets with $E_{1} \cup \ldots \cup E_{N}=[0,1)$

Now define $u_{n}=\mathscr{E}\left(w_{\infty} \mid \mathscr{B}_{n}\right)$. Then, since $\left\{w_{n}\right\}$ is uniformly bounded and $w_{n} \rightarrow w_{\infty}$ a.e.,

$$
\begin{aligned}
u_{n} & =\lim _{m \rightarrow \infty} \mathscr{E}\left(w_{N} \mid \mathscr{B}_{n}\right) \\
& =w_{n}+\left(\sum_{k=n+1}^{\infty} \mathscr{E}\left(\alpha_{k} \mid \mathscr{B}_{n}\right)\right) a \\
& =w_{n}+\theta_{n} a,
\end{aligned}
$$

where $\theta_{n} \geq 0$ is $\mathscr{B}_{n}$-measurable. Since $\left(w_{n}\right)$ is a submartingale, $\left(\theta_{n}\right)$ is a supermartingale. As $u_{n}-w_{n} \rightarrow 0$ a.e., we have $\theta_{n} \rightarrow 0$ a.e. As $\pi\left(w_{\infty}\right) \leq 1$ a.e., $\pi\left(u_{n}\right) \leq 1$ a.e. and so $\theta_{n} \leq 1$ a.e. Also $\left\|u_{n}-\theta_{n} a\right\|=\left\|w_{n}\right\|=1$. Finally observe

$$
\begin{aligned}
u_{0} & =\left(\alpha_{0}+\theta_{0}\right) a \\
& =\sum_{i=1}^{N} \lambda\left(E_{i}\right) e_{i} .
\end{aligned}
$$

Hence

$$
\begin{aligned}
\pi\left(u_{0}\right) & =\sum_{i=1}^{N} \lambda\left(E_{i}\right)=1 \\
& =\alpha_{0}+\theta_{0} .
\end{aligned}
$$

Thus $\lambda\left(E_{i}\right)=a_{i}(1 \leq i \leq N)$, and the proof is complete. 
In fact we shall not use Theorem 2.2; instead we use its "finite" version.

THEOREM 2.3. Under the same hypotheses as Theorem 2.2, given $\varepsilon>0$, there is a finite dyadic martingale $\left(v_{0}, v_{1}, \ldots, v_{m}\right)$ with

$$
\begin{array}{cc}
v_{0}(t)=a & (0 \leq t<1), \\
\left\|v_{m}(t)\right\| \leq 1+\varepsilon & (0 \leq t<1) .
\end{array}
$$

For $1 \leq n \leq m-1$, there is a positive $\mathscr{B}_{n}$-measurable function $\phi_{n}$ with $\phi_{n} \leq 1$ and

$$
\left\|v_{n}(t)-\phi_{n}(t) a\right\| \leq 1+\varepsilon \quad(0 \leq t<1) .
$$

Proof. Suppose $0<\delta_{0}<\frac{1}{2}$ is chosen so that $\left\|2 \delta_{0} a\right\|<\frac{1}{2} \varepsilon$ and $\left\|\left(1-\delta_{0}\right)^{-1}\right\|<1+\frac{1}{2} \varepsilon$ whenever $\|x\|<1$.

Let $u_{n}, \theta_{n}$ be chosen as in Theorem 2.2 and select $m$ so that

$$
\int_{0}^{1} \theta_{m}(t) d t=\delta \leq \delta_{0} .
$$

Define

$$
v_{m}=(1-\delta)^{-1}\left(u_{m}-\theta_{m} a\right)
$$

and

$$
v_{n}=\mathscr{C}\left(v_{m} \mid \mathscr{B}_{n}\right) \quad(0 \leq n \leq m) .
$$

Then $\left\|v_{m}\right\| \leq 1+\varepsilon$ and

$$
\begin{aligned}
v_{n} & =(1-\delta)^{-1}\left(u_{n}-\mathscr{E}\left(\theta_{m} \mid \mathscr{B}_{n}\right) a\right) \\
& =(1-\delta)^{-1}\left(u_{n}-\theta_{n} a\right)+(1-\delta)^{-1}\left(\theta_{n}-\mathscr{E}\left(\theta_{m} \mid \mathscr{B}_{n}\right)\right) a .
\end{aligned}
$$

Define

$$
\phi_{n}=\theta_{n}-\mathscr{E}\left(\theta_{m} \mid \mathscr{B}_{n}\right) \quad(0 \leq n \leq m) .
$$

Then $0 \leq \phi_{n} \leq \theta_{n} \leq 1$ and

$$
v_{n}-\phi_{n} a=(1-\delta)^{-1}\left(u_{n}-\theta_{n} a\right)+\delta(1-\delta)^{-1} \phi_{n} a
$$

and so

$$
\left\|v_{n}-\phi_{n} a\right\| \leq 1+\frac{1}{2} \varepsilon+\frac{1}{2} \varepsilon=1+\varepsilon .
$$

3. Main results. We now turn to the general infinite-dimensional problem.

Lemma 3.1. Suppose $X$ is an F-space with a strictly concave F-norm. Suppose $x_{0} \neq 0$ and that $x_{0} \in \operatorname{co}\{x:\|x\| \leq \delta\}$. Then there is a finite dyadic martingale $u_{n}(0 \leq n \leq m)$ with $u_{0}(t) \equiv x_{0}$, and

$$
\begin{array}{cc}
\left\|u_{m}(t)\right\| \leq 2 \delta & (0 \leq t<1) \\
\left\|u_{n}(t)\right\| \leq\left\|x_{0}\right\|+2 \delta & (0 \leq t<1,0 \leq n \leq m) .
\end{array}
$$


Proof. There exist $y_{1}, \ldots, y_{N} \in X$ with $y_{i} \neq 0 \quad(1 \leq i \leq N), \quad\left\|y_{i}\right\| \leq \delta$ and $x_{0}=$ $a_{1} y_{1}+\ldots+a_{N} y_{N}$, where $a_{i} \geq 0$ and $a_{1}+a_{2}+\ldots+a_{N}=1$.

For $0 \leq t<\infty$, define

$$
F_{i}(t)=\left\|t y_{i}\right\| /\left\|y_{i}\right\| \text {. }
$$

Then $F_{i}$ is strictly concave. Define the absolute norm on $\mathbb{R}^{N}$ by

$$
\|b\|=\sum_{i=1}^{N} F_{i}\left(\left|b_{i}\right|\right) .
$$

Now, by Theorem 2.3 , there is a finite $\mathbb{R}^{N}$-valued dyadic martingale $\left(v_{n}: 0 \leq n \leq m\right)$ with (taking $\varepsilon=1$ )

$$
\begin{gathered}
v_{0}(t) \equiv a=\left(a_{1}, \ldots, a_{N}\right) \quad(0 \leq t<1) \\
\left\|v_{m}(t)\right\| \leq 2 \quad(0 \leq t<1)
\end{gathered}
$$

and

$$
\left\|v_{n}(t)-\phi_{n}(t) a\right\| \leq 2 \quad(0 \leq t<1,0 \leq n<m),
$$

where $0 \leq \phi_{n}(t) \leq 1$. Define $T: \mathbb{R}^{N} \rightarrow X$ by

$$
T b=\sum_{i=1}^{N} b_{i} y_{i}
$$

Then

$$
\begin{aligned}
\|T b\| & \leq \sum_{i=1}^{N}\left\|b_{i} y_{i}\right\| \\
& \leq \sum_{i=1}^{N}\left\|y_{i}\right\| F_{i}\left(\left|b_{i}\right|\right) \\
& \leq \delta\|b\| .
\end{aligned}
$$

Now let $u_{n}=T v_{n}$. Then $u_{0}(t) \equiv x_{0}$ and $\left\|u_{m}(t)\right\| \leq 2 \delta$. Also

$$
\begin{aligned}
\left\|u_{n}(t)\right\| & \leq\left\|\phi_{n}(t) x_{0}\right\|+2 \delta \\
& \leq\left\|x_{0}\right\|+2 \delta
\end{aligned}
$$

Theorem 3.2. Suppose $X$ is an $F$-space with trivial dual, and that $x_{0} \in X$. Then there is a dyadic martingale $\left(u_{n}: n \geq 0\right)$ with $u_{0}(t) \equiv x_{0}$ and

$$
\max _{0 \leq t<1}\left\|u_{n}(t)\right\| \rightarrow 0 \text { as } n \rightarrow \infty .
$$

Proof. As explained in the introduction we may suppose that the $F$-norm on $X$ is strictly concave (passing to an equivalent $F$-norm does not affect (3.2.1)). The hypotheses guarantee that the convex hull of any neighborhood of zero is $X$. The construction is inductive, based on Lemma 3.1. To start the construction we may find a finite martingale 
$\left(u_{n}: 0 \leq n \leq N_{1}\right)$ so that $u_{0}(t)=x_{0},\left\|u_{N_{1}}(t)\right\| \leq \frac{1}{2}\left\|x_{0}\right\|$ and $\left\|u_{n}(t)\right\| \leq 2\left\|x_{0}\right\|\left(1 \leq n \leq N_{1}\right)$, by applying Lemma 3.1 with $\delta=\frac{1}{4}\left\|x_{0}\right\|$ if $x_{0} \neq 0$ (the case $x_{0}=0$ is trivial).

Suppose now we have defined $\left(u_{n}: 1 \leq n \leq N_{k}\right)$ so that

$$
\begin{gathered}
\left\|u_{N_{j}}(t)\right\| \leq\left(\frac{1}{2}\right)^{j}\left\|x_{0}\right\| \quad(1 \leq j \leq k), \\
\left\|u_{n}(t)\right\| \leq 2\left(\frac{1}{2}\right)^{j}\left\|x_{0}\right\| \quad\left(N_{i}<n<N_{j+1}, 1 \leq j \leq k-1\right) .
\end{gathered}
$$

We shall show how to extend to a finite dyadic martingale $\left(u_{n}: 1 \leq n \leq N_{k+1}\right)$ so that (3.2.2) and (3.2.3) hold for $j \leq k+1$ and $j \leq k$ respectively.

We have

$$
u_{N_{k}}(t)=y_{t} \quad\left(t \in D_{N_{k}, t}\right) .
$$

For each $y_{l}$, there is a finite martingale $\left(v_{n}^{l}: 0 \leq n \leq M\right)$ with

$$
\begin{gathered}
v_{0}^{l}(t)=y_{l} \quad(0 \leq t \leq 1), \\
\left\|v_{M}^{t}(t)\right\| \leq\left(\frac{1}{2}\right)^{k+1}\left\|x_{0}\right\| \quad(0 \leq t \leq 1), \\
\left\|v_{n}^{t}(t)\right\| \leq\left\|y_{l}\right\|+\left(\frac{1}{2}\right)^{k+1}\left\|x_{0}\right\| \\
\leq\left(\frac{1}{2}\right)^{k-1}\left\|x_{0}\right\| \quad(0 \leq t \leq 1,0 \leq n \leq M) .
\end{gathered}
$$

Here $M$ may be taken independent of $l$ by simply extending the martingale where necessary by adding further terms equal to the last term of the sequence.

Now let $N_{k+1}=N_{k}+M$ and define

$$
u_{N_{k}+i}=v_{1}^{l}\left(2^{N_{k}} t-l+1\right) \quad\left(t \in D_{N_{k}, l}\right) .
$$

It is now easy to verify that conditions (3.2.2) and (3.2.3) hold where applicable. Continuing in this way we clearly have (3.2.1) for the (infinite) martingale $\left(u_{n}\right)$.

The step from Theorem 3.2 to our main result is a very simple one if $X$ is a quasi-Banach space or more generally is exponentially galbed (see Turpin [8]). In such space there is a natural correspondence between curves with uniform zero derivative and dyadic martingales converging uniformly to 0 . In a general $F$-space a little more sublety is required in the proof of the main theorem.

Theorem 3.3. Suppose $X$ is an $F$-space with trivial dual and that $x_{0}, x_{1} \in X$. Then there is a curve $g:[0,1] \rightarrow X$ with $g(0)=x_{0}, g(1)=x_{1}$ and

$$
\lim _{|t-s| \rightarrow 0} \frac{g(t)-g(s)}{t-s}=0 \quad \text { uniformly for } 0 \leq s, t \leq 1 \text {. }
$$

In particular $g^{\prime}(t)=0$ for $0 \leq t \leq 1$.

Proof. It suffices to suppose $x_{0}=0$. Then there is a dyadic martingale $\left(u_{n}: n \geq 0\right)$ with

$$
\begin{aligned}
& u_{0}(t)=x_{1} \quad(0 \leq t<1), \\
& \max _{0 \leq t<1}\left\|u_{n}(t)\right\|=\varepsilon_{n} \rightarrow 0 .
\end{aligned}
$$


Choose $N_{0}=0$. Since each $u_{n}$ has finite range it is possible to choose a strictly increasing sequence of positive integers $\left(N_{k}: k \geq 1\right)$ so that

$$
\left\|2^{N_{i}-N_{k}}\left(u_{k}(t)-u_{k-1}(t)\right)\right\| \leq 2^{i-k} \varepsilon_{j}
$$

for $0 \leq j \leq k-1,0 \leq t<1$. Each $t \in[0,1)$ has a unique binary expansion

$$
t=\sum_{j=1}^{\infty} \tau_{i} 2^{-j}
$$

where each $\tau_{j}$ is zero or one and $\tau_{j}=0$ infinitely often. Now define

$$
v_{k}(t)=u_{k}\left(\sum_{i=1}^{k} \tau_{N_{i}} 2^{-i}\right)
$$
(Recall that $u_{k}$ is constant on the interval $\sum_{j=1}^{k} \tau_{N_{j}} 2^{-j} \leq t<\sum_{j=1}^{k} \tau_{N_{j}} 2^{-i}+2^{-k}$.) Then we
observe that $v_{k}$ is a $\mathscr{B}_{N_{k}}$-martingale, with

$$
\begin{gathered}
\max _{0 \leq t<1}\left\|v_{k}(t)\right\|=\varepsilon_{k}, \\
\mathscr{E}\left(v_{\mathrm{k}} \mid \mathscr{B}_{0}\right)=\int_{0}^{1} v_{k}(t) d t=\int_{0}^{1} u_{k}(t) d t=x_{1} .
\end{gathered}
$$

In fact we observe that

$$
\mathscr{E}\left(v_{k} \mid \mathscr{B}_{N_{k}-1}\right)=v_{k-1} .
$$

For $k \geq 1$ and $0 \leq t \leq 1$, we define

$$
g_{k}(t)=\int_{0}^{t} v_{k}(s) d s
$$

(the integrand is simple). Then each $g_{k}$ is continuous and from (3.3.3) we have

$$
g_{k}(t)=g_{k-1}(t) \text { if } 2^{N_{k}-1} t \in \mathbb{Z} .
$$

Now suppose that $0<t<1$ and that $2 l \leq 2^{N_{k} t}<2 l+1$, where $l$ is an integer. Then

$$
\begin{aligned}
g_{k}(t)-g_{k-1}(t) & =\int_{2 l / 2^{N_{k}}}^{t}\left(v_{k}(s)-v_{k-1}(s)\right) d s \\
& =\left(t-2 l\left(2^{-N_{k}}\right)\right)\left(v_{k}(t)-v_{k-1}(t)\right) .
\end{aligned}
$$

Equally, if $2 l+1 \leq 2^{N_{k}}<2 l+2$,

$$
g_{k}(t)-g_{k-1}(t)=\left((2 l+2) 2^{-N_{k}}-t\right)\left(v_{k}(t)-v_{k-1}(t)\right) .
$$

Combining these results, we have

$$
\begin{aligned}
\left\|g_{k}(t)-g_{k-1}(t)\right\| & \leq \max _{\substack{0 \leq 1<1\\
}}\left\|2^{-N_{k}}\left(v_{k}(t)-v_{k-1}(t)\right)\right\| \\
& =\max _{0 \leq t<1}\left\|2^{-N_{k}}\left(u_{k}(t)-u_{k-1}(t)\right)\right\| \\
& \leq 2^{-k} \varepsilon_{0} .
\end{aligned}
$$


Hence $\left(g_{k}\right)$ converges uniformly to a continuous function $g$ on $[0,1]$, and $g(0)=0$, $g(1)=x_{1}$.

Now suppose $0 \leq s<t \leq 1$. Then there is a least integer $n$ so that for some integer $l$ we have $2^{n} s \leq l<l+1 \leq 2^{n} t$. Clearly $2^{n-1} t-2^{n-1} s<2$ and $2^{n} t-2^{n} s \geq 1$. Hence $2^{-n} \leq t-s<$ $4.2^{-n}$ and $n \geq \log _{2} 1 /(t-s)$.

Now suppose $N_{k-1} \leq n<N_{k}$, where $1 \leq k<\infty$. Suppose $l_{1}$ is the least integer not less than $2^{n} s$ and $l_{2}$ is the greatest integer not greater than $2^{n} t$. Then

$$
\begin{aligned}
2^{n}\left(g_{k-1}(t)-g_{k-1}\left(l_{2} 2^{-n}\right)\right) & =\left(2^{n} t-l_{2}\right) v_{k-1}\left(l_{2} 2^{-n}\right), \\
2^{n}\left(g_{k-1}\left(l_{1} 2^{-n}\right)-g_{k-1}(s)\right) & =\left(l_{1}-2^{n} s\right) v_{k-1}\left(l_{1} 2^{-n}\right), \\
2^{n}\left(g_{k-1}\left(i 2^{-n}\right)-g_{k-1}\left((i-1) 2^{-n}\right)\right) & =v_{k-1}\left((i-1) 2^{-n}\right) .
\end{aligned}
$$

Hence

$$
\left\|2^{n}\left(g_{k-1}\left(l_{2} 2^{-n}\right)-g_{k-1}\left(l_{1} 2^{-n}\right)\right)\right\| \leq\left(l_{2}-l_{1}\right) \varepsilon_{k-1}
$$

and

$$
\left\|2^{n}\left(g_{k-1}(t)-g_{k-1}(s)\right)\right\| \leq\left(l_{2}-l_{1}+2\right) \varepsilon_{k-1} .
$$

However $l_{2}-l_{1} \leq 2^{n}(t-s)<4$ so that $l_{2}-l_{1}+2 \leq 5$. Hence

$$
\left\|2^{n}\left(g_{k-1}(t)-g_{k-1}(s)\right)\right\| \leq 5 \varepsilon_{k-1} .
$$

Now

$$
2^{n}\left(g_{k}(t)-g_{k-1}(t)\right)=2^{n-N_{k}} \rho\left(v_{k}(t)-v_{k-1}(t)\right),
$$

where $0 \leq \rho \leq 1$, by (3.3.4) and (3.3.5). Hence

$$
\left\|2^{n}\left(g_{k}(t)-g_{k-1}(t)\right)\right\| \leq \varepsilon_{k}+\varepsilon_{k-1} .
$$

A similar inequality holds for $s$.

If $r>k$

$$
2^{n}\left(g_{r}(t)-g_{r-1}(t)\right)=2^{n-N_{r}} \rho\left(v_{r}(t)-v_{r-1}(t)\right),
$$

where $0 \leq \rho \leq 1$, and so

$$
\begin{aligned}
\left\|2^{n}\left(g_{r}(t)-g_{r-1}(t)\right)\right\| & \leq\left\|2^{N_{k}-N_{r}}\left(v_{r}(t)-v_{r-1}(t)\right)\right\| \\
& \leq \max _{0 \leq t<1}\left\|2^{N_{k}-N_{r}}\left(u_{r}(t)-u_{r-1}(t)\right)\right\| \\
& \leq 2^{k-r} \varepsilon_{k}
\end{aligned}
$$

by (3.3.2). Hence

$$
\left\|2^{n}\left(g(t)-g_{k}(t)\right)\right\| \leq\left(\sum_{r>k} 2^{k-r}\right) \varepsilon_{k}=\varepsilon_{k} .
$$

A similar inequality holds for $s$. 
Combining (3.3.6), (3.3.7) and (3.3.8) and the similar results for $s$ we obtain

$$
\left\|2^{n}(g(t)-g(s))\right\| \leq 7 \varepsilon_{k-1}+4 \varepsilon_{k}
$$

and hence

$$
\left\|\frac{g(t)-g(s)}{t-s}\right\| \leq 7 \varepsilon_{k-1}+4 \varepsilon_{k}
$$

where $N_{k}>\log _{2} 1 /(t-s)$. Hence $g$ has the properties specified in the theorem.

Every $F$-space $X$ has a unique maximal linear subspace with trivial dual; this subspace is closed. Let us call this maximal subspace the core of $X$. If core $(X)=\{0\}$, it does not necessarily follow that $X$ has a separating dual; for a detailed investigation of related ideas see Ribe [3]. We conclude with a simple corollary.

Corollary 3.4. Suppose $X$ is an F-space and $x \in X$. In order that there exists a curve $g:[0,1] \rightarrow X$ with $g(0)=0, g(1)=x$ and $g^{\prime}(t)=0$ for $0 \leq t \leq 1$ it is necessary and sufficient that $x \in \operatorname{core}(X)$.

Proof. If $x \in \operatorname{core}(X)$ the existence of $g$ is given by Theorem 3.3. Suppose conversely such a $g$ exists and let $Y$ be the closed linear span of $\{g(t): 0 \leq t \leq 1\}$. Suppose $\phi$ is a continuous linear functional on $Y$. Then $(\phi \circ g)^{\prime}(t)=0(0 \leq t \leq 1)$ and hence by the Mean Value Theorem $\phi(g(t))=0 \quad(0 \leq t \leq 1)$. Thus $\phi=0$ and so $Y \subset \operatorname{core}(X)$; in particular $x \in \operatorname{core}(X)$.

\section{REFERENCES}

1. C. Bessaga, A. Pelczyński and S. Rolewicz, Some properties of the norm in F-spaces, Studia Math. 16 (1957), 183-192.

2. N. J. Kalton, An F-space with trivial dual where the Krein-Milman theorem holds, Israel J. Math. 36 (1980), 41-50.

3. M. Ribe, On the separation properties of the duals of general topological vector spaces, Ark. Mat. 9 (1971), 279-302. 255-266.

4. J. W. Roberts, A compact convex set with no extreme points. Studia Math. 60 (1977),

5. J. W. Roberts, Pathological compact convex sets in the spaces $L_{p}, 0 \leq p \leq 1$, The Altgeld Book (University of Illinois, 1976).

6. S. Rolewicz, O funkcjach o pochodnej zero, Wiadom. Math. (2) 3 (1959), 127-128.

7. S. Rolewicz, Metric linear spaces (PWN Warsaw, 1972).

8. P. Turpin, Convexités dans les espaces vectoriels topologiques généraux, Dissertationes Math. (Rozprawy Mat.) 131 (1976).

Department of Mathematics

University of MisSouri-Columbia

Columbia

MIsSOURI 65211

U.S.A. 